\title{
NLRP3 rs35829419 polymorphism is associated with increased susceptibility to multiple diseases in humans
}

\author{
Q. Zhang, H.W. Fan, J.Z. Zhang, Y.M. Wang and H.J. Xing \\ Department of Orthopedics, China Japan Union Hospital of Jilin University, \\ Changchun, China \\ Corresponding author: H.J. Xing \\ E-mail: xinghongjian123@126.com \\ Genet. Mol. Res. 14 (4): 13968-13980 (2015) \\ Received May 2, 2015 \\ Accepted July 31, 2015 \\ Published October 29, 2015 \\ DOI http://dx.doi.org/10.4238/2015.October.29.17
}

ABSTRACT. Using a meta-analysis framework, we investigated the association between the NLRP3 rs35829419 polymorphism and increased susceptibility to diverse diseases in humans. Relevant published studies were identified through a comprehensive and systematic electronic search, using the following scientific literature databases: Science Citation Index, the Cochrane Library, PubMed, Embase, CINAHL, Current Contents Index, Chinese Biomedical, the Chinese Journal Full-Text, and the Weipu Journal. Statistical analysis of data extracted from the selected high quality studies was performed using the Version 12.0 STATA software. A total of 13 case-control studies met our stringent inclusion and exclusion criteria for the present meta-analysis. These 13 high quality studies contained relevant information on 7719 patients with various diseases and 7094 healthy controls. Our meta-analysis results showed that the NLRP3 gene rs35829419 C>A polymorphism was associated with a significantly increased risk of developing multiple diseases in humans under 5 genetic models (all $P<0.05$ ). Data stratification and subgroup analysis based on the disease type revealed that rs35829419 C>A carriers displayed a markedly increase susceptibility to leprosy, colorectal cancer, HIV-1 infection, 
rheumatoid arthritis, abdominal aortic aneurysms, inflammatory bowel disease, ulcerative colitis, and atopic dermatitis. In summary, our metaanalysis results revealed the first identified strong correlation between the NLRP3 rs35829419 polymorphism and increased susceptibility to various diseases in humans.

Key words: NLRP3; Genetic polymorphism; Leprosy; Colorectal cancer; HIV-1 infection; Rheumatoid arthritis

\section{INTRODUCTION}

The NACHT domain, leucine rich repeats (LRR), and pyrin domain (PYD)-containing protein 3 (NLRP3), formerly known as cryopyrin, is a member of the NOD-like receptor (NLR) family (Choi and Ryter, 2014). NLRP3 is the major player in the multi-protein complex known as the inflammasome, and interacts with several adaptor proteins such as apoptosis-associated speck-like protein (ASC), pro-caspase-1, and other proteins that contain caspase activation and recruitment domains (CARD) (Verma et al., 2008). In humans, NLRP3 is encoded by the NOD-like receptor family, pyrin domain containing 3 gene (NLRP3), also known as the cold induced autoinflammatory syndrome 1 (CIAS1) gene (Lamkanfi and Kanneganti, 2010). NLRP3 is primarily expressed by dendritic cells and macrophages and is rapidly activated by pathogen-associated molecular patterns such as viral DNAs, peptidoglycans, bacterial lipopolysaccharides and toxins, as well as stress or danger signals such as UV radiation and reactive oxygen species (Wanderer, 2008). The NLRP3 complex shows a strong response to cytidine phosphate-guanosine DNA, ssRNA, and dsRNA in bacteria and viruses, thus detecting microbial pathogens and mounting an immune response (Villani et al., 2009a). The NLRP3 gene is approximately $32.9 \mathrm{~kb}$ in length, contains 9 exons, and maps to the long arm of chromosome 1q44 (Kastbom et al., 2008; Zhang et al., 2011). Within the NLRP3 gene, approximately 60 single nucleotide polymorphisms (SNPs) have been identified, and the most prevalent polymorphisms among these are rs35829419 (Q705K), rs10754558, rs4612666, rs4925648, and rs10925019 (Schoultz et al., 2009), based on the SNP genotype data available from the US National Institute of Mental Health bipolar disorder (NIMH-BP) database dbGaP. In general, NLRP3 rs35829419 polymorphisms are associated with various inflammatory diseases such as rheumatoid arthritis, Crohn's disease, and celiac disease (Pontillo et al., 2011; Pontillo et al., 2012a; Tan et al., 2013), and the NLRP3 polymorphism Q705K in particular is tightly linked to autoimmune disorders such as atopic dermatitis and systemic lupus erythematosus (Roberts et al., 2010; Tan et al., 2013) and to neuropathies such as late-onset Alzheimer's disease (Ben Hamad et al., 2012). It is well known that inflammatory diseases are characterized by excessive production of nuclear factor-kB (NF-kB)-dependent cytokines such as interleukin (IL)-1 $\beta$, and that the NLRP3 gene polymorphism Q705K is a gain-of-function variant that leads to hyperactive inflammasomes. The heightened activity of inflammasome sowing to NLRP3 gene polymorphism Q705Klikely constitutively cleaves pro-IL-1 $\beta$ leading to the overproduction of bioactive IL-1 $\beta$, which participates in the host response to injury and infection as a proinflammatory cytokine (Roberts et al., 2011; Pontillo et al., 2012a). The pathogenic mechanism involving NLRP3 rs35829419 SNP is dramatically similar in the diverse disease settings discussed above (Roberts et al., 2010; Ben Hamad et al., 2012), and accumulated evidence suggests that the Q705K polymorphism promotes a disease state by causing a hyper-sensitive response to 
pathogen-associated molecular patterns and sustained activation of inflammation (Villani et al., 2009b; Kastbom et al., 2013). However, in direct contrast, other studies have shown a protective role for the NLRP3 polymorphism Q705K; therefore, the clinical relevance of this polymorphism remains uncertain (Hitomi et al., 2009; Ben Hamad et al., 2012). In order to address this issue, we performed a comprehensive meta-analysis to evaluate the association of the NLRP3 Q705K gene polymorphism with the progression of a variety of human diseases of high clinical value.

\section{MATERIAL AND METHODS}

\section{Search strategy}

To identify relevant published studies, a comprehensive literature search was conducted without language restriction using the following computerized bibliographic databases: Science Citation Index, the Cochrane Library, PubMed, Embase, CINAHL, Current Contents Index, Chinese Biomedical; the Chinese Journal Full-Text, and the Weipu Journal (last updated search in September 2014). Only literature published in the English and Chinese languages were selected for this meta-analysis. The highly sensitive search strategy included the search terms: ("NLRP3 protein, human" or "NLRP3" or "PYRIN-containing Apaf1-like protein 1" or "NLR family, pyrin domain containing 3" or "PYPAF1" or "CIAS1" or "cryopyrin") and ("polymorphism, genetic" or "polymorphism" or "polymorphisms" or "variants" or "SNP" or "mutation" or "genetic variants"). Cross-references of relevant articles were manually searched to identify other relevant studies.

\section{Inclusion and exclusion criteria}

The retrieved studies were assessed for their suitability to this meta-analysis using the following inclusion criteria: 1) case-control studies reported the correlation between the single nucleotide polymorphism (SNP) in NLRP3 (rs35829419) and the risk of human diseases; 2) blood samples were obtained from patients with confirmed diagnosis for each disease type; 3 ) the reports contained sufficient original data and information on the NLRP3 rs35829419 polymorphism; 4) distributions of NLRP3 genotype frequencies met Hardy-Weinberg equilibrium (HWE) in the controls; and 5) only the latest complete study was considered when the extracted studies were published by the same authors. Studies that did not meet the inclusion criteria were excluded, along with studies published in non-English or non-Chinese language.

\section{Study quality and data extraction}

The Newcastle-Ottawa Scale (NOS) criteria was employed to assess the methodological quality of the included trials (Zintzaras and loannidis, 2005). The assessment of study suitability and quality was performed by two reviewers based on the inclusion/exclusion criteria. A standardized data form was utilized to collect the descriptive information in each included study. Collected information included the first author, publication year or submission year, country, journal, ethnicity, language, diseases type, source of controls, confirmation of diagnosis, and demographic variables of subjects, genotype frequencies, HWE test, and detection method. Disagreements during data collection were resolved by reexamination of all items and by discussion with a third investigator. 


\section{Statistical analysis}

STATA statistical software (Version 12.0, Stata Corporation, College Station, TX, USA) was applied for statistical analyses. The effect sizes representing the unadjusted ORs for the presence of the NLRP3SNP in patients and controls were calculated. A Z test was conducted to assess the significance of the overall effect size. Pooled ORs were generated for comparisons using the allelic (C allele vs $A$ allele), dominant $(C C+C A$ vs $A A)$, recessive $(C C$ vs $C A+A A)$, homozygous ( $C C$ vs $A A$ ), and heterozygous ( $C C$ vs $C A$ ) models. The heterogeneity between all the studies was evaluated with Cochran's $Q$-statistic $\left(P<0.05\right.$ was considered significant) and the $l^{2}$ test $(0 \%$, no heterogeneity; $100 \%$, maximal heterogeneity).A random effects model was used in instances of significant heterogeneity $\left(P<0.05\right.$ or $l^{2}$ test exhibited $>50 \%$ ); otherwise, a fixed effect model was applied (Alhopuro et al., 2009; Kastbom et al., 2010). Additionally, a sensitivity analysis was performed to evaluate whether removal of any single study could influence the overall outcomes. Further, Egger's linear regression test and a funnel plot were applied to assess publication bias (Pontillo et al., 2010; Girardelli et al., 2012).

\section{RESULTS}

\section{Description of included studies}

A total of 361 potentially eligible articles were identified from the literature. After exclusion of 3 duplicate studies and 219 irrelevant articles, 140 articles remained for further evaluation. Subsequently, 124 articles were excluded, and16 articles were examined closely for qualitative analysis. This resulted in elimination of 3 studies that had insufficient information; finally, 13 casecontrol studies were incorporated into this meta-analysis that contained a total of 7719 patients diagnosed with diverse diseases and 7094 healthy controls (Hitomi et al., 2009; Villani et al., 2009a,b; Roberts et al., 2010; Roberts et al., 2011; Ben Hamad et al., 2012; Carlstrom, 2012; Pontillo et al., 2012a,b; Ungerback, 2012; Kastbom et al., 2013; Pontillo et al., 2013; Varghese et al., 2013). The various diseases in the included studies were myocardial infarction, Alzheimer's disease, leprosy, ankylosing spondylitis, colorectal cancer (CRC), HIV-1 infection, systemic lupus erythematosus, malignant mesothelioma, rheumatoid arthritis (RA), abdominal aortic aneurysms (AAA), inflammatory bowel disease (IBD), ulcerative colitis (UC), Crohn's disease (CD), type 1 diabetes, celiac disease, and atopic dermatitis. Among the 13 studies, 1 study each was performed in Asian and African populations, and the remaining 11 studies were conducted in Caucasian populations. The healthy controls were drawn from population-basedsources. The detection techniques employed in the studies included in this meta-analysis were polymerase chain reaction/ligase detection reaction $(P C R-L D R)(N=1)$ and TaqMan assay $(N=12)$. The baseline characteristics of the 13 studies are shown in Table 1.

\section{Quantitative data synthesis}

In this meta-analysis, most of the studies shown in the bivariate boxplot figure fell into the central area of the shaded section, but two studies fell outside this area, indicating heterogeneity among the studies (Figure 1). We then investigated the associations between the allele and genotype frequencies of the NLRP3 rs35829419 polymorphism and the susceptibilities to related 
diseases. The meta-analysis revealed that, out of the 16 diseases reported in the selected studies, the NLRP3 gene (rs35829419) polymorphism conferred significantly increased susceptibility to 12 , in both the allelic and dominant models (allele model: $\mathrm{OR}=3.74,95 \% \mathrm{Cl}=1.91-7.30, \mathrm{P}<$ 0.001 ; dominant allele: $\mathrm{OR}=13.37,95 \% \mathrm{Cl}=3.90-45.82, \mathrm{P}<0.001$ ) (Figure 2). The results of this meta-analysis implied that carriers of the NLRP3 rs35829419 C>A polymorphic variant were at an elevated risk of developing multiple diseases in comparison to human subjects without this variant, based on the pooled OR (all P<0.05) (Table 2).

Table 1. Baseline characteristics and methodological quality of all included studies.

\begin{tabular}{|c|c|c|c|c|c|c|c|c|c|c|}
\hline \multirow[t]{2}{*}{ First author } & \multirow[t]{2}{*}{ Country } & \multirow[t]{2}{*}{ Disease } & \multicolumn{2}{|c|}{ Sample size } & \multicolumn{2}{|c|}{ Gender (M/F) } & \multicolumn{2}{|c|}{ Age (years) } & \multirow{2}{*}{$\begin{array}{l}\text { Genotyping } \\
\text { method }\end{array}$} & \multirow{2}{*}{$\begin{array}{l}\text { NOS } \\
\text { score }\end{array}$} \\
\hline & & & Patients & Controls & Patients & Controls & Patients & Controls & & \\
\hline Varghese (2013) & Sweden & Myocardial infarction & 491 & 911 & $349 / 142$ & $653 / 258$ & - & - & TaqMan assay & 7 \\
\hline $\operatorname{Tan}(2013)$ & China & Alzheimer's disease & 1133 & 1159 & $6464 / 669$ & $518 / 641$ & $79.9 \pm 8.1$ & $74.5 \pm 6.3$ & PCR-LDR & 8 \\
\hline Pontillo (2013) & Brazil & Leprosy & 467 & 380 & $325 / 142$ & $252 / 128$ & $40.7 \pm 17.5$ & $35.9 \pm 10.7$ & TaqMan assay & 7 \\
\hline Kastbom (2013) & Sweden & Ankylosing spondylitis & 492 & 793 & $338 / 154$ & $396 / 397$ & 51 & 54 & TaqMan assay & 7 \\
\hline Ungerback (2012) & Sweden & Colorectal cancer & 348 & 806 & - & - & - & - & TaqMan assay & 6 \\
\hline Pontillo (2012) & Brazil & HIV-1 infection & 150 & 158 & $80 / 70$ & $72 / 86$ & $34.0 \pm 12.4$ & $29.0 \pm 7.8$ & TaqMan assay & 6 \\
\hline Pontillo (2012) & Brazil & Systemic lupus erythematosus & 144 & 158 & $8 / 136$ & $83 / 75$ & $37.0 \pm 11.9$ & $34.7 \pm 11.7$ & TaqMan assay & 6 \\
\hline Girardelli (2012) & Italy & Malignant mesothelioma & 134 & 256 & $112 / 22$ & $118 / 138$ & $66.0 \pm 13.0$ & $42.0 \pm 7.0$ & TaqMan assay & 6 \\
\hline \multirow[t]{2}{*}{ Ben Hamad (2012) } & France & Rheumatoid arthritis & 100 & 100 & - & - & $32.0 \pm 10.0$ & - & TaqMan assay & 6 \\
\hline & Tunisia & Rheumatoid arthritis & 141 & 191 & - & $58 / 133$ & $39.5 \pm 20.0$ & 43 & TaqMan assay & 6 \\
\hline Roberts (2011) & New Zealand & Abdominal aortic aneurysms & 1238 & 731 & $987 / 251$ & $469 / 262$ & $74.2 \pm 7.9$ & $69.9 \pm 6.7$ & TaqMan assay & 8 \\
\hline \multirow[t]{3}{*}{ Roberts (2010) } & New Zealand & Inflammatory bowel disease & 982 & 517 & $410 / 572$ & $226 / 291$ & - & $49(37-65)$ & TaqMan assay & 8 \\
\hline & New Zealand & Ulcerative colitis & 475 & 517 & $228 / 247$ & $226 / 291$ & $39(28-53)$ & $49(37-65)$ & TaqMan assay & 7 \\
\hline & New Zealand & Crohn's disease & 507 & 517 & $182 / 325$ & $226 / 291$ & $32(23-50)$ & $49(37-65)$ & TaqMan assay & 7 \\
\hline \multirow[t]{3}{*}{ Pontillo (2010) } & Brazil & Type-1 diabetes & 196 & 192 & $94 / 102$ & $90 / 102$ & $7.3 \pm 3.4$ & $7.1 \pm 4.8$ & TaqMan assay & 6 \\
\hline & Brazil & Celiac disease & 59 & 192 & $21 / 38$ & $90 / 102$ & $10.0 \pm 4.0$ & $7.1 \pm 4.8$ & TaqMan assay & 6 \\
\hline & Brazil & Atopic dermatitis & 165 & 192 & $84 / 81$ & $90 / 102$ & $7.0 \pm 5.0$ & $7.1 \pm 4.8$ & TaqMan assay & 6 \\
\hline Schoultz (2009) & Sweden & Crohn's disease & 497 & 742 & $257 / 244$ & $384 / 356$ & $28(10-90)$ & - & TaqMan assay & 7 \\
\hline
\end{tabular}

$\mathrm{M}=$ male; $\mathrm{F}=$ female; $\mathrm{NOS}=$ Newcastle-Ottawa scale; $\mathrm{PCR}-\mathrm{LDR}=$ polymerase chain reaction-ligase detection reaction.

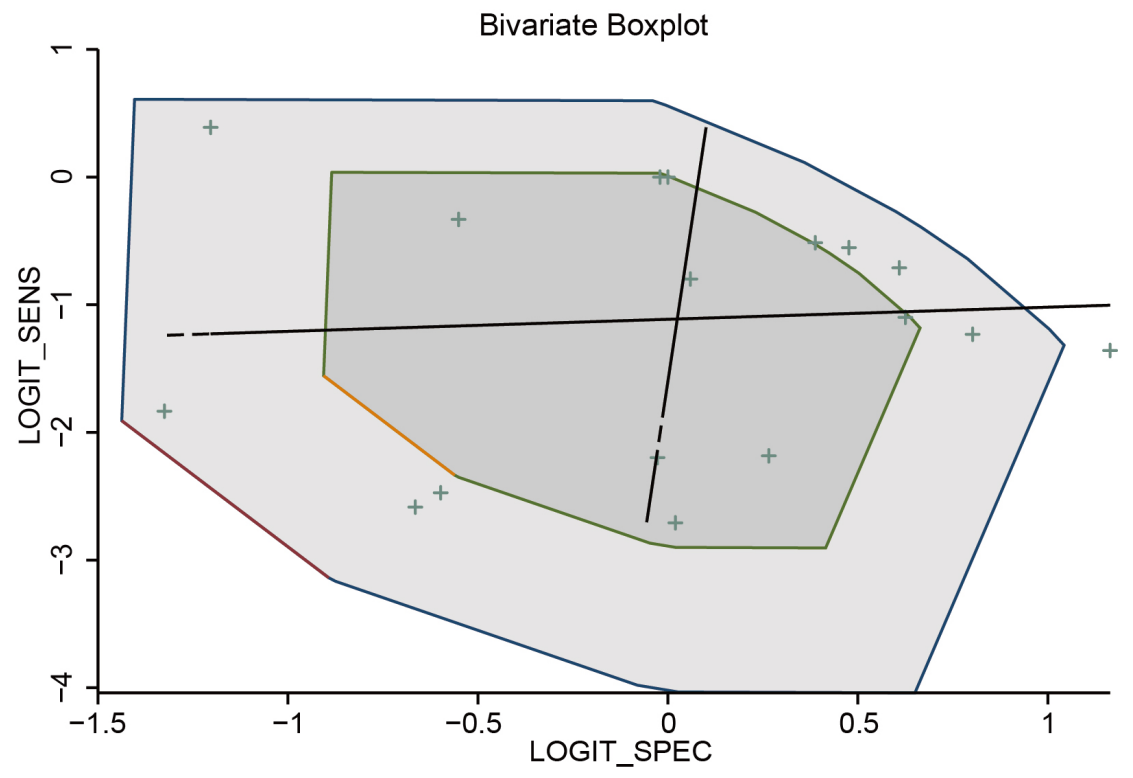

Figure 1. Test for heterogeneity in the included studies by a bivariate boxplot. 


\begin{tabular}{|c|c|c|c|}
\hline $\begin{array}{l}\text { A } \\
\text { Included study }\end{array}$ & $\begin{array}{l}19 \mathrm{C}>\mathrm{A} \\
\text { us } \mathrm{M} \text { allele) }\end{array}$ & OR $(95 \% \mathrm{Cl})$ & Weight \% \\
\hline Varghese GP (2013) & & $1.11(0.84,1.46)$ & 5.99 \\
\hline Tan MS (2013) & & $14.71(0.84,257.67)$ & 2.87 \\
\hline Pontillo A (2013) & & $2.26(1.29,3.96)$ & 5.80 \\
\hline Kastbom A (2013) & & $1.08(0.78,1.49)$ & 5.97 \\
\hline Ungerback J (2012) & & $1.54(1.10,2.15)$ & 5.96 \\
\hline Pontillo A (2012a) & & $9.25(2.78,30.82)$ & 5.06 \\
\hline Pontillo A (2012b) & & $2.09(0.94,4.68)$ & 5.57 \\
\hline Girardelli M (2012) & & $1.61(0.77,3.34)$ & 5.64 \\
\hline Ben Hamad M (2012a) & & $3.15(1.38,7.23)$ & 5.54 \\
\hline Ben Hamad M (2012b) & $\rightarrow$ & $18.86(9.18,38.74)$ & 5.66 \\
\hline Roberts RL (2011) & & $2.42(1.88,3.11)$ & 6.00 \\
\hline Roberts RL (2010a) & $\leftarrow$ & $23.55(18.44,30.07)$ & 6.00 \\
\hline Roberts RL (2010b) & $\leftarrow$ & $21.55(15.69,29.59)$ & 5.97 \\
\hline Roberts RL (2010c) & $\leftarrow$ & $25.78(18.51,35.92)$ & 5.96 \\
\hline Pontillo A (2010a) & & $1.02(0.25,4.11)$ & 4.80 \\
\hline Pontillo A (2010b) & & $1.21(0.57,2.61)$ & 5.61 \\
\hline Pontillo A (2010c) & & $6.81(3.21,14.41)$ & 5.63 \\
\hline Schoultz I (2009) & & $1.13(0.82,1.57)$ & 5.97 \\
\hline $\begin{array}{l}\text { Heterogeneity test }\left(l^{2}=97.7 \%, P<0.001\right) \\
Z \text { test }(Z=3.86, P<0.001)\end{array}$ & & $3.74(1.91,7.30)$ & 100.00 \\
\hline Random effects analysis & & & \\
\hline
\end{tabular}

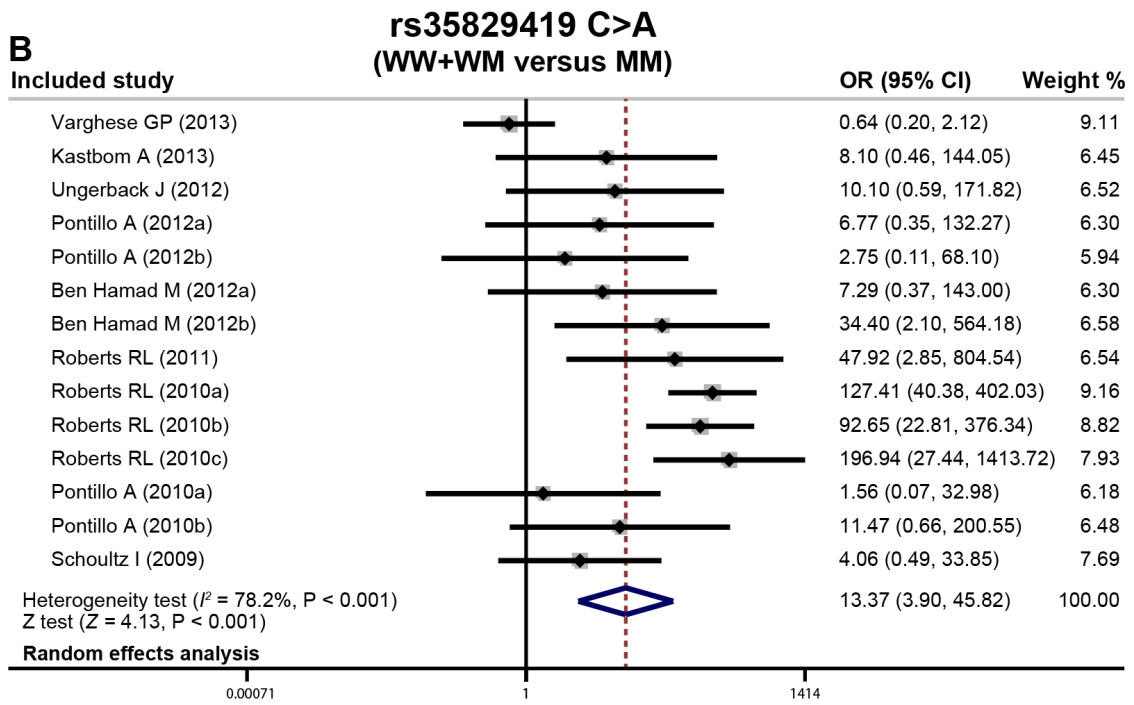

Figure 2. Forest plots for the influences of the NLRP3 genetic polymorphism and various human diseases under the allelic and dominant models ( $\mathrm{A}, \mathrm{W}$ allele vs $\mathrm{M}$ allele; $\mathrm{B}, \mathrm{WW}+\mathrm{WM}$ vs $\mathrm{MM}$ ).

In subgroup analysis stratified by disease type, the results showed an elevated risk of related diseases in carriers of the NLRP3 rs35829419 C>A polymorphism in leprosy, CRC, RA, $A A A, I B D, U C$, and $A D$ in the majority of subgroups (Figure 3 ). 


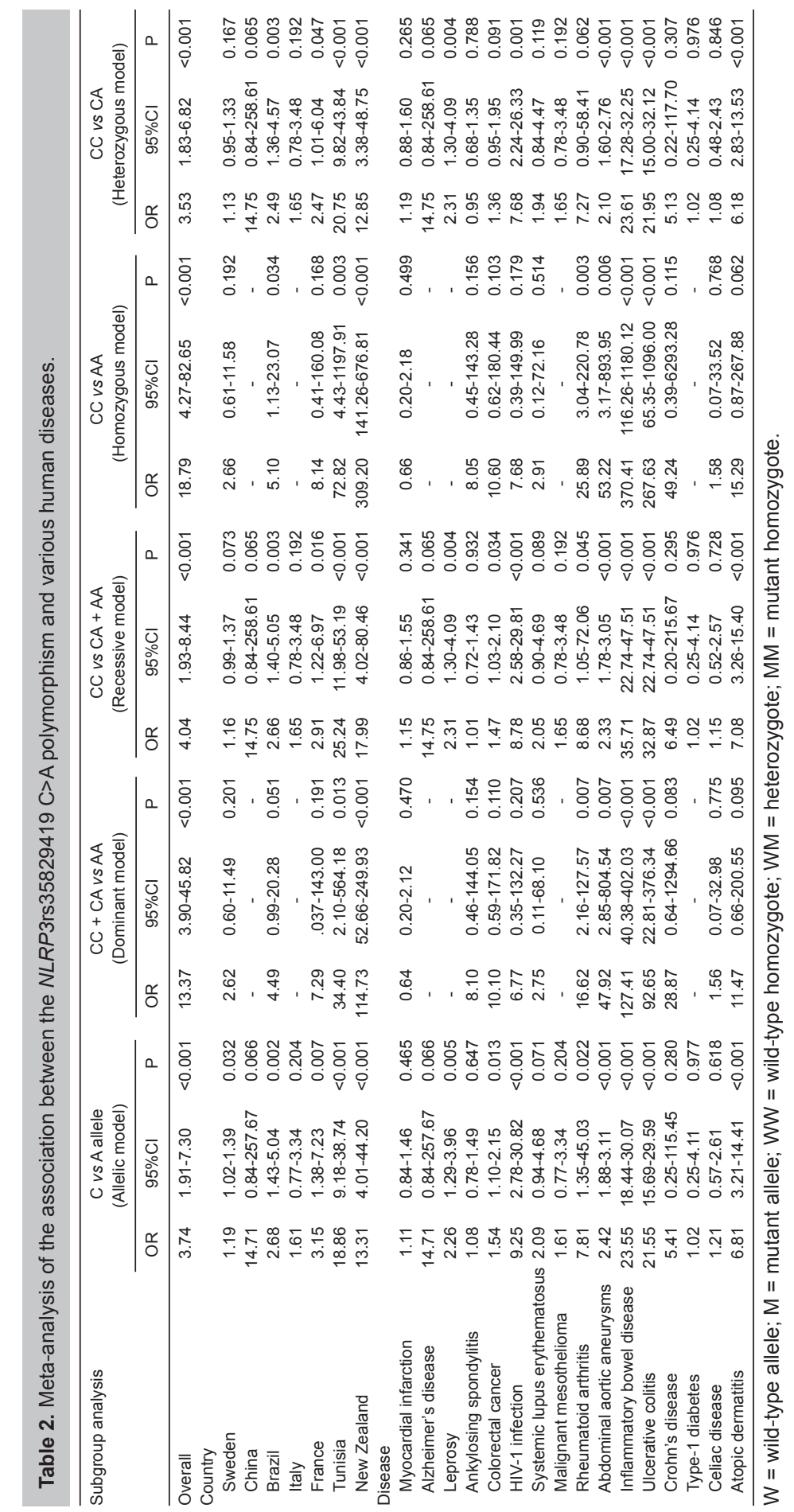


Meta-regression analysis results confirmed that no single factor was the main source of heterogeneity (Figure 4 and Table 3). Sensitivity analysis was conducted to determine whether the study conclusions were affected by any single study; the finding suggested that no single study had an effect on the pooled ORs (Figure 5). Furthermore, the Egger's regression test and funnel plot showed symmetrical distribution for the associations of the NLRP3 rs35829419 C>A polymorphism and the risk of various diseases (all $P>0.05$ ) (Figure 6).

A

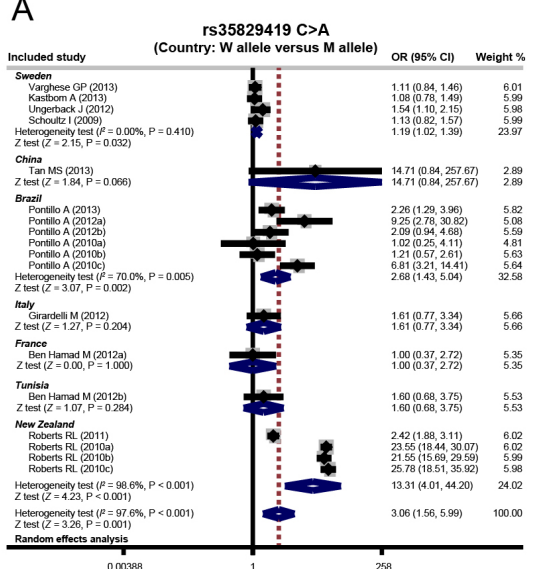

C

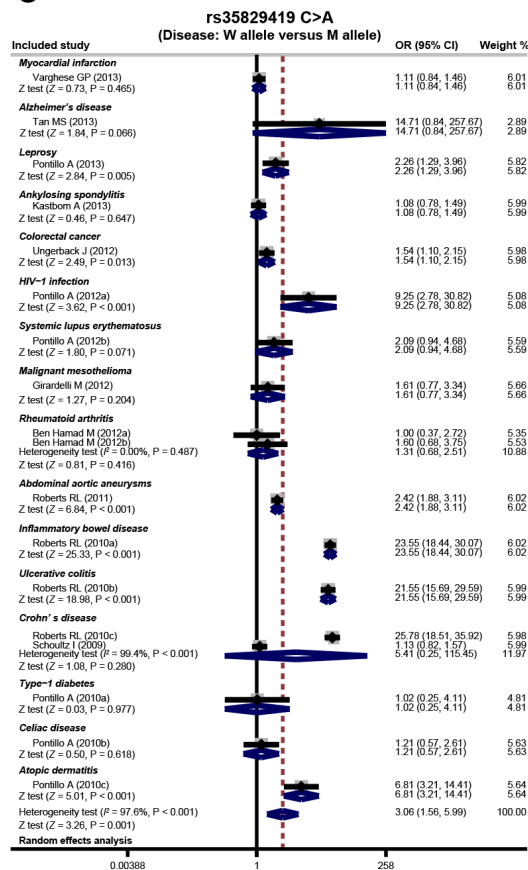

B

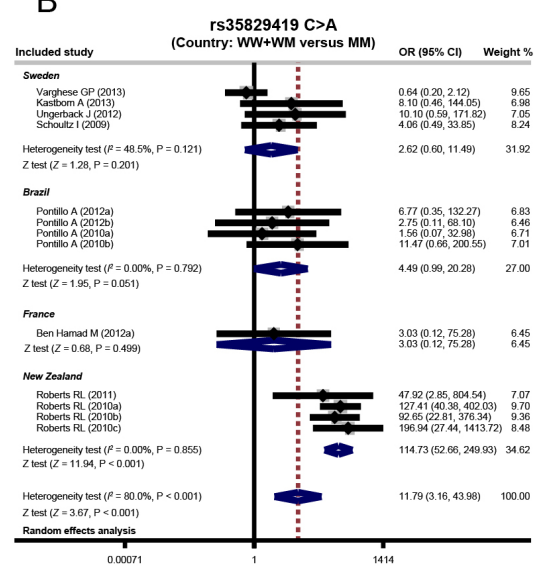

D

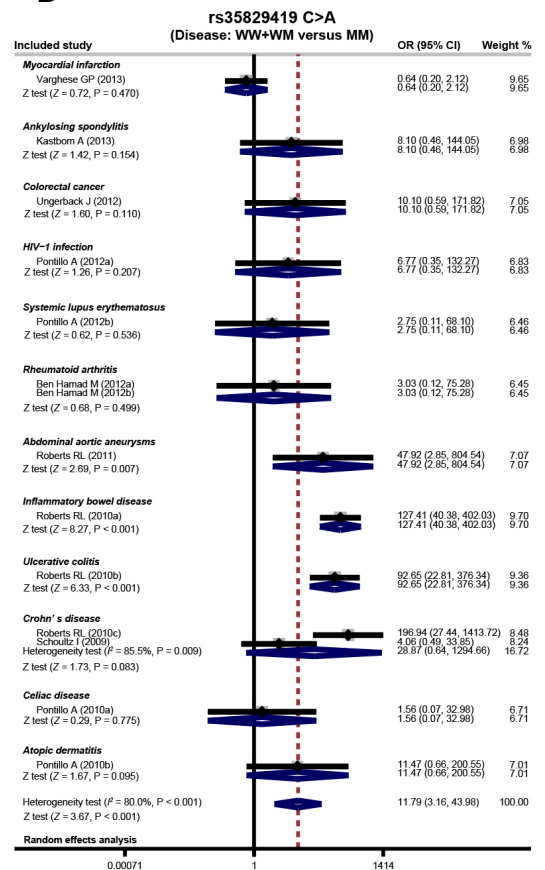

Figure 3. Subgroup analyses for the influences of the NLRP3 genetic polymorphism and various human diseases under the allelic and dominant models (A, country: W allele vs $M$ allele; $B$, country: $W W+W M$ vs $M M$; C, disease: $W$ allele vs $\mathrm{M}$ allele; $\mathrm{D}$, disease: $\mathrm{WW}+\mathrm{WM}$ vs $\mathrm{MM})$. 

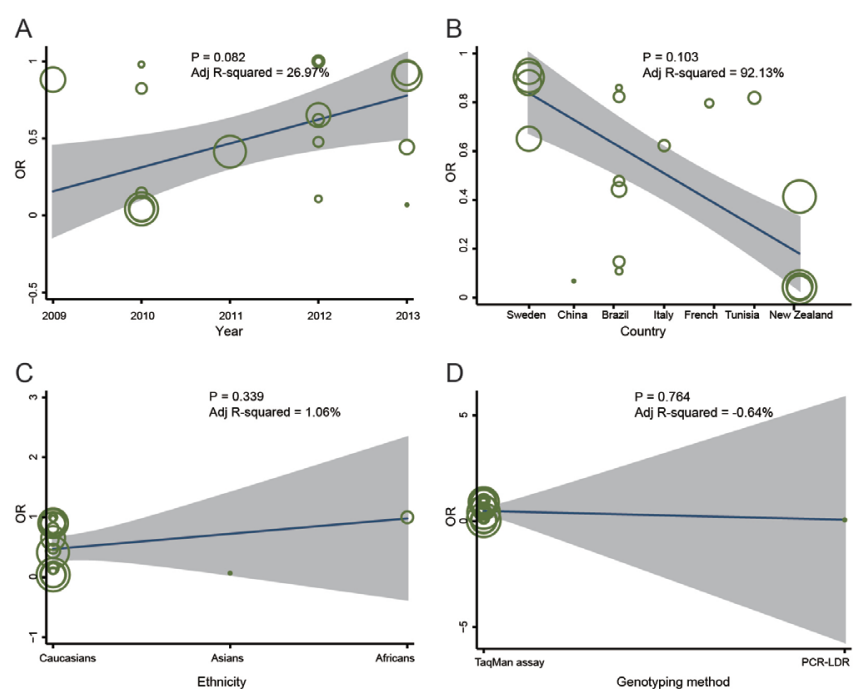

Figure 4. Univariate meta-regression analysis of potential sources of heterogeneity. PCR-LDR, polymerase chain reaction-ligation detection reaction. (A, year; $B$, country, $C$, ethnicity, $D$, genotyping method).

A
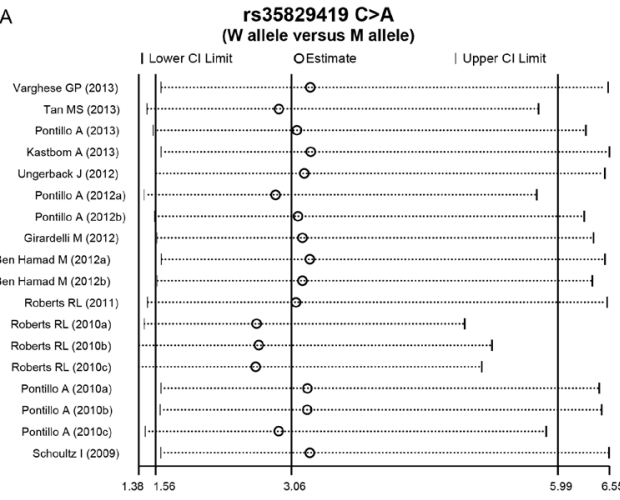

rs35829419 C>A

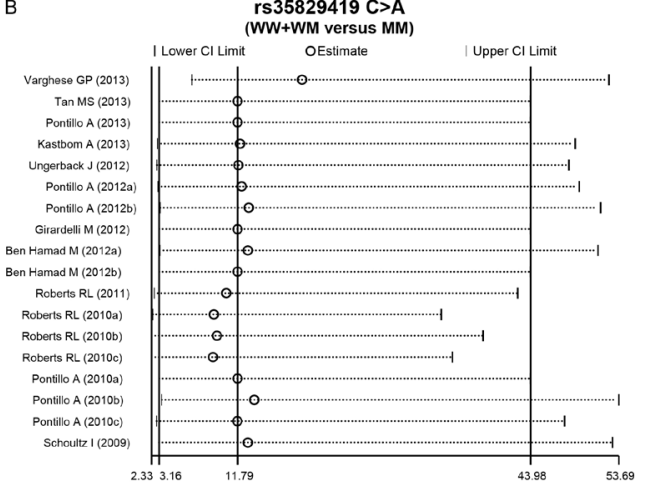

Figure 5. Sensitivity analyses for the influences of the $N L R P 3$ genetic polymorphism and various human diseases under the allelic and dominant models (A, W allele vs $M$ allele; B, WW+WM vs MM). 
A

rs35829419 C>A
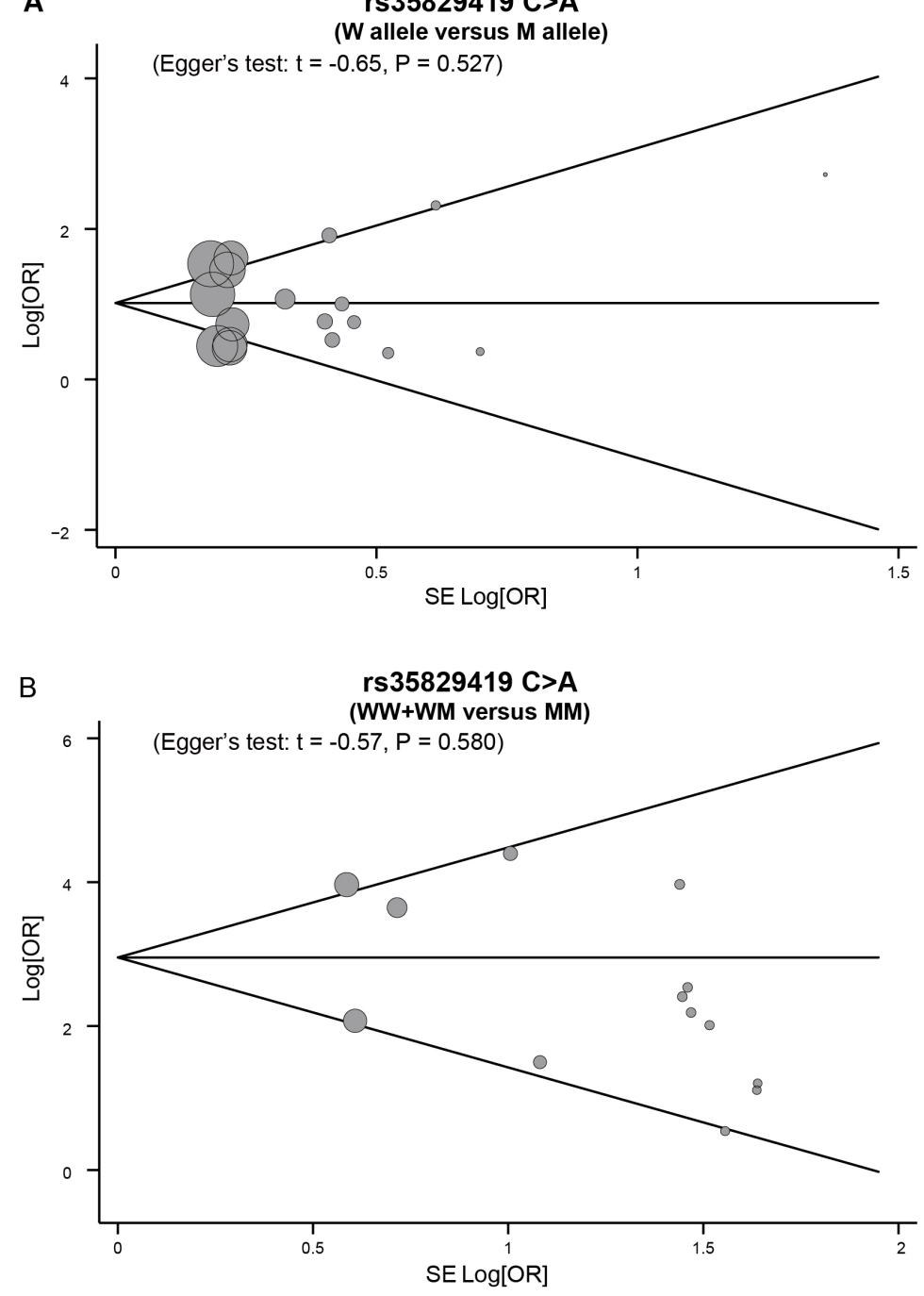

Figure 6. Egger's regression test of the relationships between the NLRP3 genetic polymorphism and various human diseases under the allelic and dominant models ( $\mathrm{A}, \mathrm{W}$ allele $v s \mathrm{M}$ allele; $\mathrm{B}, \mathrm{WW}+\mathrm{WM}$ vs $\mathrm{MM}$ ).

\begin{tabular}{|c|c|c|c|c|c|c|}
\hline \multirow[t]{2}{*}{ Heterogeneity factor } & \multirow[t]{2}{*}{ Coefficient } & \multirow[t]{2}{*}{ SE } & \multirow[t]{2}{*}{$\mathrm{t}$} & \multirow[t]{2}{*}{ P (adjusted) } & \multicolumn{2}{|c|}{$95 \% \mathrm{Cl}$} \\
\hline & & & & & LL & UL \\
\hline Year & 0.031 & 0.049 & 0.65 & 0.939 & -0.072 & 0.135 \\
\hline Country & -0.105 & 0.023 & -4.48 & 0.108 & -0.155 & -0.054 \\
\hline Ethnicity & 0.345 & 0.186 & 1.86 & 0.258 & -0.057 & 0.746 \\
\hline Genotyping method & -1.038 & 1.478 & -0.70 & 0.910 & -4.226 & 2.150 \\
\hline
\end{tabular}

SE = standard error; $\mathrm{LL}=$ lower limit; UL = upper limit. 


\section{DISCUSSION}

We used a meta-analysis based approach to examine the association between the NLRP3 rs35829419 gene polymorphism and the development of inflammatory diseases. The results of our meta-analysis suggest a close link between the NLRP3 rs35829419 gene polymorphism and the disease pathogenesis in leprosy, CRC, RA, AAA, IBD, UC, and AD.

The NLRP3 protein contains NACHT, LRR, and PYD domains. The NACHT domain is a nucleotide binding domain, while LRR is a leucine-rich repeat domain used for detecting microbial pathogens, and PYD is useful for protein-protein interaction with the PYD of apoptosis-associated speck-like protein (ASC), which also contains a CARD domain; together these domains are important in assembly of the inflammasome multi-protein complex for mediating the inflammatory process and apoptosis signaling in response to various stimuli (Ji et al., 2012). Through PYD-PYD interactions, NLRP3 recruits ASC, which in turn leads to the assembly ofpro-caspase-1 through CARD-CARD interactions, and its subsequentactivation allows the maturation and release of IL$1 \beta$, causing widespread inflammatory reactions (Cummings et al., 2010).

$N L R P 3$ gene polymorphisms are associated with various inflammatory diseases such as CRC, AAA, and CD (Hitomi et al., 2009). The rs35829419 variant is located in exon 3 of the NLRP3 gene, which is close to the NACHT domain where most NLRP3 gene mutations are also found (Pontillo et al., 2010). In this meta-analysis, we determined that the minor A allele of rs35829419 conferred protection against CRC, the mechanisms of which might be related to the interaction of NLRP3 with caspase-1 in inflammasomes, promoting release of pro-inflammatory cytokines such as IL-1 $\beta$ through the inflammatory NF-kB pathway, which participates in apoptosis (Varghese et al., 2013). On the other hand, in IBD, including UC and CD, and in abdominal aortic aneurysms, the protective mechanism is thought to involve a genetic interaction between the rs2043211 polymorphism in the CARD8 gene and rs35829419 in NLRP3 (Villani et al., 2009b). The $T$ allele in rs2043211 of theCARD8 gene produces a severely truncated CARD8 protein; as CARD8 is a constituent protein of the NLRP3 inflammasome, CARD8 truncation further results in promoting pro-IL-1 $\beta$ production and there by preventing the pathological inflammation typical in IBD (Roberts et al., 2011). Based on previous studies and our results in this meta-analysis, we propose that the NLRP3 gene rs35829419 polymorphism affects a variety of inflammatory diseases, positively or negatively, primarily through its role in the inflammasome complex and its protein-protein interactions with other members of the complex. Similar conclusions were reached by Varghese et al. (2013), who showed that the minor A allele in the rs35829419 polymorphism was protective against myocardial infarction in women through increased production of IL- $1 \beta$, which had a cooperative beneficial effect in combination with the female hormone estrogen (Carlstrom, 2012).

In order to investigate other influencing factors, subgroup analysis based on country showed a strong link in both the allelic and dominant models between the NLRP3 gene polymorphism and the studied diseases in New Zealand and Tunisian populations. However, in other countries the variants had no detectable disease associations, which might be due to environment, genetic background, or lifestyle differences. Clear relationships between rs35829419 and RA, IBD, UC, and AAA were observed in both the allelic and dominant models. Our results are partly in agreement with previous studies in that the NLRP3 gene rs35829419polymorphism was found to be linked to several inflammatory diseases, suggesting that the NLRP3 genetic polymorphism is a critical predisposing factor in the development of various human diseases. Therefore, detection of 
the NLRP3 rs35829419 gene polymorphism might permit early prediction of the risks for various human diseases, and might provide a basis for designing treatments as well as for additional studies on the susceptibility to disease.

The limitations of our study should be also mentioned. First, only one SNP (rs35829419) was investigated, and the involvement of additional genomic loci in the disease outcomes cannot be ruled out. Additionally, there were a total of 16 different types of diseases involved in the present meta-analysis that might be caused by different etiological factors; therefore, the clinical applications of our final results might be limited. Thirdly, the current meta-analysis only included trials published in English and Chinese, and nearly all (11/13) of the studies were performed in Caucasians with only 1 in Asians and 1 in Africans. Further studies are needed to better represent other ethnic groups to clarify any ethnic differences in the frequency of effect of the NLRP3 rs35829419 polymorphism. Furthermore, heterogeneity existed in this meta-analysis and complete data was not available from all of the included studies. The study in Asians did not conform to HWE, which influenced the accuracy of the results, and should have been excluded.

Taken together, our findings suggest that the NLRP3 rs35829419 genetic polymorphism is involved in the progression of several human diseases, especially of leprosy, CRC, RA, AAA, $I B D, U C$, and $A D$. Further clinical studies and functional analyses are required to investigate the mechanisms underlying the genetic interactions between the NLRP3 gene with other genomic loci, and the susceptibility to various diseases in humans.

\section{Conflicts of interest}

The authors declare no conflict of interest.

\section{REFERENCES}

Alhopuro P, Klimenko T and Aittomäki K (2009). Fever from the cold - familial cold autoinflammatory syndrome. Duodecim 125: 542-545.

Ben Hamad M, Cornelis F, Marzouk S, Chabchoub G, et al. (2012). Association study of CARD8 (p.C10X) and NLRP3 (p.Q705K) variants with rheumatoid arthritis in French and Tunisian populations. Int. J. Immunogenet. 39: 131-136.

Carlström M, Ekman AK, Petersson S, Söderkvist P, et al. (2012). Genetic support for the role of the NLRP3 inflammasome in psoriasis susceptibility. Exp. Dermatol. 21: 932-937.

Choi AJ and Ryter SW (2014). Inflammasomes: molecular regulation and implications for metabolic and cognitive diseases. Mol. Cells 37: 441-448.

Cummings JR, Cooney RM, Clarke G, Beckly J, et al. (2010). The genetics of NOD-like receptors in Crohn's disease. Tissue Antigens 76: 48-56.

Girardelli M, Maestri I, Rinaldi RR, Tognon M, et al. (2012). NLRP1 polymorphisms in patients with asbestos-associated mesothelioma. Infect. Agent. Cancer 7: 25.

Hitomi Y, Ebisawa M, Tomikawa M, Imai T, et al. (2009). Associations of functional NLRP3 polymorphisms with susceptibility to food-induced anaphylaxis and aspirin-induced asthma. J. Allergy Clin. Immunol. 124: 779-785.e6.

Ji X, Hou Z, Wang T, Jin K, et al. (2012). Polymorphisms in inflammasome genes and risk of coal workers' pneumoconiosis in a Chinese population. PLoS One 7: e47949.

Kastbom A, Verma D, Eriksson P, Skogh T, et al. (2008). Genetic variation in proteins of the cryopyrin inflammasome influences susceptibility and severity of rheumatoid arthritis (the Swedish TIRA project). Rheumatology 47: 415-417.

Kastbom A, Johansson M, Verma D, Söderkvist $\mathrm{P}$, et al. (2010). CARD8 p.C10X polymorphism is associated with inflammatory activity in early rheumatoid arthritis. Ann. Rheum. Dis. 69: 723-726.

Kastbom A, Klingberg E, Verma D, Carlsten H, et al. (2013). Genetic variants in CARD8 but not in NLRP3 are associated with ankylosing spondylitis. Scand. J. Rheumatol. 42: 465-468.

Lamkanfi M and Kanneganti TD (2010). NIrp3: an immune sensor of cellular stress and infection. Int. J. Biochem. Cell Biol. 
42: $792-795$

Pontillo A, Brandao L, Guimaraes R, Segat L, et al. (2010). Two SNPs in NLRP3 gene are involved in the predisposition to type-1 diabetes and celiac disease in a pediatric population from northeast Brazil. Autoimmunity 43: 583-589.

Pontillo A, Vendramin A, Catamo E, Fabris A, et al. (2011). The missense variation Q705K in CIAS1/NALP3/NLRP3 gene and an NLRP1 haplotype are associated with celiac disease. Am. J. Gastroenterol. 106: 539-544.

Pontillo A, Girardelli M, Kamada AJ, Pancotto JA, et al. (2012a). Polimorphisms in inflammasome genes are involved in the predisposition to systemic lupus erythematosus. Autoimmunity 45: 271-278.

Pontillo A, Oshiro TM, Girardelli M, Kamada AJ, et al. (2012b). Polymorphisms in inflammasome' genes and susceptibility to HIV-1 infection. J. Acquir. Immune Defic. Syndr. 59: 121-125.

Pontillo A, Laurentino W, Crovella S and Pereira AC (2013). NLRP1 haplotypes associated with leprosy in Brazilian patients. Infect. Genet. Evol. 19: 274-279.

Roberts RL, Topless RK, Phipps-Green AJ, Gearry RB, et al. (2010). Evidence of interaction of CARD8 rs2043211 with NALP3 rs35829419 in Crohn's disease. Genes Immun. 11: 351-356.

Roberts RL, Van Rij AM, Phillips LV, Young S, et al. (2011). Interaction of the inflammasome genes CARD8 and NLRP3 in abdominal aortic aneurysms. Atherosclerosis 218: 123-126.

Schoultz I, Verma D, Halfvarsson J, Törkvist L, et al. (2009). Combined polymorphisms in genes encoding the inflammasome components NALP3 and CARD8 confer susceptibility to Crohn's disease in Swedish men. Am. J. Gastroenterol. 104: 1180-1188

Tan MS, Yu JT, Jiang T, Zhu XC, et al. (2013). NLRP3 polymorphisms are associated with late-onset Alzheimer's disease in Han Chinese. J. Neuroimmunol. 265: 91-95.

Ungerbäck J, Belenki D, Jawad ul-Hassan A, Fredrikson M, et al. (2012). Genetic variation and alterations of genes involved in NFkappaB/TNFAIP3- and NLRP3-inflammasome signaling affect susceptibility and outcome of colorectal cancer. Carcinogenesis 33: 2126-2134.

Varghese GP, Fransén K, Hurtig-Wennlöf A, Bengtsson T, et al. (2013). Q705K variant in gene confers protection against myocardial infarction in female individuals. Biomed. Rep. 1: 879-882.

Verma D, Lerm M, Blomgran Julinder R, Eriksson P, et al. (2008). Gene polymorphisms in the NALP3 inflammasome are associated with interleukin-1 production and severe inflammation: relation to common inflammatory diseases? Arthritis Rheum. 58: 888-894.

Villani AC, Lemire M, Fortin G, Louis E, et al. (2009a). Common variants in the NLRP3 region contribute to Crohn's disease susceptibility. Nat. Genet. 41: 71-76.

Villani AC, Lemire M, Louis E, Silverberg MS, et al. (2009b). Genetic variation in the familial Mediterranean fever gene (MEFV) and risk for Crohn's disease and ulcerative colitis. PLoS One 4: e7154.

Wanderer AA (2008). Ischemic-reperfusion syndromes: biochemical and immunologic rationale for IL-1 targeted therapy. Clin. Immunol. 128: 127-132.

Zhang AQ, Zeng L, Gu W, Zhang LY, et al. (2011). Clinical relevance of single nucleotide polymorphisms within the entire NLRP3 gene in patients with major blunt trauma. Crit. Care 15: R280.

Zintzaras E and loannidis JP (2005). Heterogeneity testing in meta-analysis of genome searches. Genet. Epidemiol. 28: 123-137. 